\title{
Erratum
}

Virchows Archiv A Pathol Anat (1989) 415:437-445

\section{Collagen secretion granules in reactive stromal myofibroblasts, with preliminary observations on their occurrence in spindle cell tumours}

B.P. Eyden

Department of Histopathology, Christie Hospital and Holt Radium Institute, Manchester, United Kingdom

On page 444, paragraph 3 , lines 7 and 8 should read:

"..., in non-neoplastic cells the elaboration of collagen as discrete fibrils is of somewhat more restricted occurrence: ...". 